\title{
Cell Size and Succinate Dehydrogenase Activity of Different Types of Fibers in Different Regions of the Tibialis Anterior Muscle in Mice and Rats
}

\author{
Chiyoko Hirofuji ${ }^{1,2}$, Toshiaki Nakatani ${ }^{3}$, Akihiko Ishihara ${ }^{1}$, Masaki Tanaka $^{4}$, \\ Kazuo Itoh $^{5}$, Minoru Itoh ${ }^{1}$, Shigeru Katsuta ${ }^{5}$ and Yasuhiko Ibata ${ }^{4}$ \\ ${ }^{1}$ Laboratory of Neurochemistry, Faculty of Integrated Human Studies, Kyoto University, Sakyo-ku, Kyoto 606-8501, \\ ${ }^{2}$ Laboratory of Exercise Physiology, Osaka Gakuin College, Suita 564-8511, ${ }^{3}$ Human Performance and Exercise \\ Prescription, Tenri University, Tenri 632-0071, 'Department of Anatomy and Neurobiology, Kyoto Prefectural \\ University of Medicine, Kamigyo-ku, Kyoto 602-0841 and ${ }^{5}$ Graduate School of Integrated Science and Art, \\ University of East Asia, Shimonoseki 751-0807
}

Received June 23, 2000; accepted September 14, 2000

\begin{abstract}
Cross-sectional areas (CSAs) and succinate dehydrogenase (SDH) activities of different types of fibers in deep, middle, and superficial regions of the tibialis anterior muscle in mice and rats were determined using quantitative histochemistry. In both mice and rats, mean CSAs of fibers were in the rank order of type Ila < type IIx < type Ilb, while mean SDH activities of fibers were in the rank order of type Ila $>$ type IIx $>$ type Ilb, irrespective of the muscle region. In mice, mean CSAs of the corresponding type of fibers were in the rank order of deep $<$ middle $<$ superficial, while there were no differences in the mean SDH activity of the fibers among the three muscle regions. In rats, type lla and type llx fibers in
\end{abstract}

the deep region had smaller CSAs and higher SDH activities than the corresponding type of fibers in the middle region, while mean CSAs of type Ilb fibers were in the rank order of deep $<$ middle $=$ superficial and mean SDH activities of the fibers were in the rank order of deep $>$ middle $=$ superficial. Muscle fibers in mice had smaller CSAs and higher SDH activities than the corresponding type of fibers in rats. These results indicate speciesspecific patterns and regional differences in the CSA and the SDH activity of fibers in the muscle even within the same fiber type defined by myosin heavy chain isoform expressions.

Key words: Cross-sectional area, Mouse, Rat, Succinate dehydrogenase activity, Tibialis anterior muscle

\section{Introduction}

Mammalian skeletal muscles consist of heterogeneous types of fibers according to the differences in their myosin adenosine triphosphatase (mATPase) activities [2, 3] or myosin heavy chain (MHC) isoform expressions $[6,13$, $28,29,33]$. Enzyme- or immunohistochemical-techniques that permit single muscle fiber analyses have revealed a distinctive profile of the enzyme activity among fibers of a given fiber type $[1,15,20,21]$. Previous studies [18, 19, $22,27,31]$ demonstrated that wide variations in the oxidative enzyme activity of fibers in the muscle are observed even within the same fiber type defined by

Correspondence to: Dr. Akihiko Ishihara, Laboratory of Neurochemistry, Faculty of Integrated Human Studies, Kyoto University, Sakyo-ku, Kyoto 606-8501, Japan.
mATPase activities. However, it would be expected that smaller-sized fibers, especially those fibers in the deep region of the muscle, have higher oxidative enzyme activities than larger-sized fibers in the superficial region even within the same fiber type defined by mATPase activities or MHC isoform expressions, because supplies of oxygen and substrates for oxidative energy metabolism from capillaries, which are located close to the membrane and are highly dense in the deep region, are more plentiful in smaller-sized fibers [35]. This would be true for different species having different muscle fiber sizes, because small animals have smaller-sized fibers in the muscle than large ones. Therefore, it is suggested that fibers in the muscles of smaller animals have higher oxidative enzyme activities than those of larger ones even within the same fiber type. The present study examined cell sizes and oxidative enzyme activities of different types of fibers, as defined 
by their MHC isoform expressions, in the deep, middle, and superficial regions of the tibialis anterior muscle in mice and rats.

\section{Materials and Methods}

\section{Animals}

Ten-week-old ICR male mice ( $\mathrm{n}=8$, body weight $38.5-42.0 \mathrm{~g})$ and Wistar male rats $(\mathrm{n}=8$, body weight $292.5-315.5 \mathrm{~g}$ ) were used in the present study. All experiments were approved by the Institutional Animal Care Committee at the University and conducted under the Guide for the Care and Use of Laboratory Animals published by the Office of Science and Health Reports of the USA National Institutes of Health, Bethesda, Maryland, USA.

\section{Tissue processing}

The right tibialis anterior muscles were removed under sodium pentobarbital anesthesia $(50 \mathrm{mg} / \mathrm{kg}$ body weight, i.p.). Thereafter, the animals were sacrificed by overdose of sodium pentobarbital. The muscles were placed on cork board at their in vivo length and quickly frozen in isopentane cooled with liquid nitrogen. Serial transverse sections, $10 \mu \mathrm{m}$ thick, of the mid-belly of the muscle were cut in a cryostat set at $-20^{\circ} \mathrm{C}$. The sections were brought to room temperature, and air-dried for $30 \mathrm{~min}$.

Immunohistochemical stainings were performed on serial sections using monoclonal antibodies specific to MHC isoforms: type I (Novocastra Laboratories), type IIa (German Collection of Microorganisms and Cell Cultures), type IIa+type IIx (Developmental Studies Hybridoma Bank, University of Iowa), and type IIb (German Collection of Microorganisms and Cell Cultures). The sections were incubated with an optimal dilution of the primary antibody overnight at $4^{\circ} \mathrm{C}$. The avidin-biotin immunohistochemical procedure was used for the localization of MHC specific primary antibody binding according to the instructions for a PK-6102 kit (Vector Laboratories) for IgG (type I and type IIa) or an AK-5010 kit for IgM (type IIa + type IIx and type IIb). In control experiments, the primary antibody was omitted. The sections were incubated with the secondary antibody for $60 \mathrm{~min}$ for both IgG and IgM at room temperature. After incubation with the antibody, the sections were dehydrated in graded series of ethanols, passed through xylene and then coverslipped. Phosphate-buffered saline was used as the buffer for the IgG class primary antibodies and Tris (hydroxymethyl) aminomethane-buffered saline was used as the buffer for the IgM class primary antibodies.

The sections were incubated for the demonstration of mATPase and succinate dehydrogenase (SDH) $[4,16,17]$. For determination of the alkali preincubation mATPase activity, the following procedures were employed: 1) preincubation for $15 \mathrm{~min}$ at room temperature in $75 \mathrm{mM}$ glycine, $50 \mathrm{mM} \mathrm{CaCl}_{2}$, and $75 \mathrm{mM} \mathrm{NaCl}$ in distilled water, adjusted to $\mathrm{pH} 10.4$ with $\mathrm{NaOH} ; 2$ ) washing in 5 changes of distilled water; 3) incubation for $45 \mathrm{~min}$ at $37^{\circ} \mathrm{C}$ in $2.8 \mathrm{mM}$ ATPase, $50 \mathrm{mM} \mathrm{CaCl}_{2}$, and $75 \mathrm{mM} \mathrm{NaCl}$ in distilled water, adjusted to $\mathrm{pH} 9.4$ with $\mathrm{NaOH}$; 4) washing in 5 changes of distilled water; 5) immersion for $3 \mathrm{~min}$ in $1 \% \mathrm{CaCl}_{2} ; 6$ ) washing in 5 changes of distilled water; 7) immersion for $3 \mathrm{~min}$ in $2 \% \mathrm{CoCl}_{2} ; 8$ ) washing in 5 changes of distilled water; 9) immersion for 1 min in $1 \%\left(\mathrm{NH}_{4}\right)_{2} \mathrm{~S}$; 10) washing in 5 changes of distilled water; and 11) dehydration in graded series of ethanols, passed through xylene and then coverslipped. Basically, the same protocol was used for the acid preincubation conditions. However, acid preincubation was carried out for $5 \mathrm{~min}$ at room temperature in $50 \mathrm{mM}$ sodium acetate and $30 \mathrm{mM}$ sodium barbital in distilled water, adjusted to $\mathrm{pH} 4.5$ and 4.3 with $\mathrm{HCl}$.

The SDH activity was determined in an incubation medium containing $100 \mathrm{mM}$ phosphate buffer $(\mathrm{pH} 7.6$ ), $0.9 \mathrm{mM}$ sodium azide, $0.9 \mathrm{mM}$ 1-methoxyphenazine methylsulfate, $1.5 \mathrm{mM}$ nitroblue tetrazolium, $5.6 \mathrm{mM}$ EDTA disodium salt, and $48 \mathrm{mM}$ succinate disodium salt. The SDH activities at $45 \mathrm{~min}$ of incubation time were used for comparison among muscle fibers of different types, because the activities of the muscle fibers showed a plateau after $45 \mathrm{~min}$ of incubation. The reaction was stopped by multiple washings in distilled water and the sections were dehydrated in graded series of ethanols, passed through xylene and then coverslipped. Histochemical control sections, in which either succinate disodium salt or nitroblue tetrazolium was excluded from the incubation medium, showed no positive SDH staining.

\section{Tissue analysis}

The cross-sectional areas (CSAs) and the SDH activities of fibers in the deep (close to the bone), middle (between the deep and superficial regions), and superficial (away from the bone) regions of the muscle were examined using a computer-assisted image processing system (Fig. 1) $[16,17]$. Approximately 50 fibers were sampled from each region in the muscle. The sections were digitized as gray scale images. Each pixel was quantified as one of 256 gray levels. A gray level value of zero was equivalent to $100 \%$ transmission of light and that of 255 was equivalent to $0 \%$ transmission of light. The mean optical density (OD) value of all pixels within a fiber was determined using a calibration tablet which has 21 steps of gradient density ranges and corresponding diffused density values [9-11].

\section{Statistics}

A two-way analysis of variance (ANOVA) was used to evaluate the influence of region and species. When the main effects or the interactions between region and species were significant based on the ANOVA analyses, the Scheffé post hoc procedures were used to determine whether CSAs or SDH activities were significantly different from each other. 


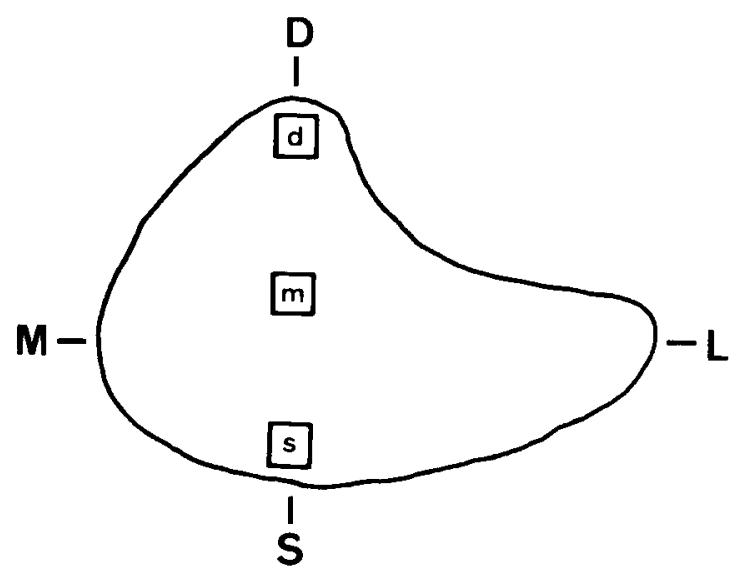

Fig. 1. Cross-sectional view showing the position of the deep (d), middle $(\mathrm{m})$, and superficial (s) regions of the mouse and rat tibialis anterior muscle. D, deep direction; S, superficial direction; $\mathrm{M}$, medial direction; $\mathbf{L}$, lateral direction.

\section{Results}

\section{Muscle fiber type classification based on MHC isoform expressions}

Fig. 2 shows serial transverse sections of the deep region in the mouse $(\mathrm{A}-\mathrm{F})$ and rat $(\mathrm{G}-\mathrm{L})$ tibialis anterior muscles. Type I fibers marked as 1 show positive stainings for type I MHC antibody and were observed only in the deep region of the rat muscle $(\mathrm{G})$. Type IIa and type IIb fibers marked as 2 and 3 show positive stainings for type IIa $\mathrm{MHC}$ antibody ( $\mathrm{B}$ and $\mathrm{H}$ ) and type IIb MHC antibody (D and J), respectively. Type IIx fibers marked as 4 show positive stainings for type IIa + type IIx MHC antibody (C and I), but negative stainings for type IIa MHC antibody (B and $\mathrm{H}$ ).

\section{CSAs and SDH activities of muscle fibers}

In both mice and rats, mean CSAs of fibers were in the rank order of type IIa $<$ type II $<$ type IIb, while mean SDH activities of fibers were in the rank order of type IIa $>$ type IIx $>$ type IIb, irrespective of the muscle region (Figs. 3-5 and Table 1). Type I fibers were observed only in the deep region of the rat muscle. There were no significant differences in the mean CSA between type I and type IIa fibers in the deep region, while type I fibers had lower SDH activities than type IIa fibers and higher SDH activities than type IIx fibers (Figs. 4, 5 and Table 1).

In mice, mean CSAs of the corresponding type of fibers were in the rank order of deep $<$ middle $<$ superficial, while there were no significant differences in the mean
SDH activity of the fibers among the three muscle regions (Figs. 3, 5 and Table 2). In rats, type IIa and type IIx fibers in the deep region had smaller CSAs and higher SDH activities than the corresponding type of fibers in the middle region (Figs. 4, 5 and Table 2). Mean CSAs of type IIb fibers were in the rank order of deep $<$ middle $=$ superficial, while mean SDH activities of the fibers were in the rank order of deep $>$ middle = superficial.

Muscle fibers in mice had smaller CSAs and higher $\mathrm{SDH}$ activities than the corresponding type of fibers in rats $(p<0.001)$.

\section{Discussion}

The distinction of type I, type IIa, and type IIb fibers by Brook and Kaiser [2, 3] was derived from differences in the $\mathrm{pH}$ lability of mATPase activity. Thereafter, a fourth fiber type, named type IIx, has been classified [6, 33]. It is well established that there is a close association between the enzyme histochemical classification of fiber types and the expression of $\mathrm{MHC}$ isoforms; the fast subtypes, type IIa, type IIb, and type IIx contain the correspondingly named MHC isoforms MHC IIa (type IIa), MHC IIb (type IIb), and MHC IIx (type IIx), whereas the slow type I contains MHC I (type I), although it is difficult to distinguish fibers containing type IIx $\mathrm{MHC}$ isoform by the method using enzyme histochemistry [14, 29, 33].

In rats, type IIa fibers have higher oxidative enzyme activities than type IIb fibers in the fast muscles, i.e. plantaris, tibialis anterior, and extensor digitorum longus muscles [16, 17]. In addition, type IIa fibers have smaller sizes than type IIb fibers in these muscles $[16,17]$. Previous studies $[23,24]$, however, reported an inverse pattern of SDH and size distribution of type IIa and type IIb fibers in the mouse tibialis anterior muscle, indicating that type IIa fibers have lower oxidative enzyme activities and larger sizes than type IIb fibers in the muscle. In the present study using monoclonal antibodies against $\mathrm{MHC}$ isoforms, we have found different $\mathrm{mATPase}$ activity patterns following preincubation at $\mathrm{pH} 4.5$ for type IIa and type IIb fibers in the mouse tibialis anterior muscle compared with the rat tibialis anterior muscle. In mouse tibialis anterior muscle, type IIa and type IIb fibers showed positive and negative stainings following preincubation at $\mathrm{pH} 4.5$, respectively ( $\mathrm{E}$ in Fig. 2). In contrast, type IIa and type IIb fibers in the rat tibialis anterior muscle showed negative and positive stainings following preincubation at $\mathrm{pH} 4.5$, respectively ( $\mathrm{K}$ in Fig. 2). We examined other mouse muscles and found that only the tibialis anterior muscle showed positive

Fig. 2. Serial transverse sections of 10 -week-old mouse $(\mathbf{A}-\mathbf{F})$ and rat $(\mathbf{G}-\mathbf{L})$ tibialis anterior muscles. The sections are stained with the monoclonal antibodies against specific myosin heavy chain isoforms ( $\mathbf{A}$ and $\mathbf{G}, \mathbf{I} ; \mathbf{B}$ and $\mathbf{H}$, IIa; $\mathbf{C}$ and I, IIa + IIx; D and $\mathbf{J}$, IIb) and for myosin adenosine triphosphatase activity following preincubation at $\mathrm{pH} 4.5$ ( $\mathbf{E}$ and $\mathbf{K}$ ) and for succinate dehydrogenase activity (F and $\mathbf{L}$ ). 1 , type I; 2, type IIa; 3, type IIb; 4, type IIx. Scale bar in A indicates $50 \mu \mathrm{m}$. 



Fig. 2 

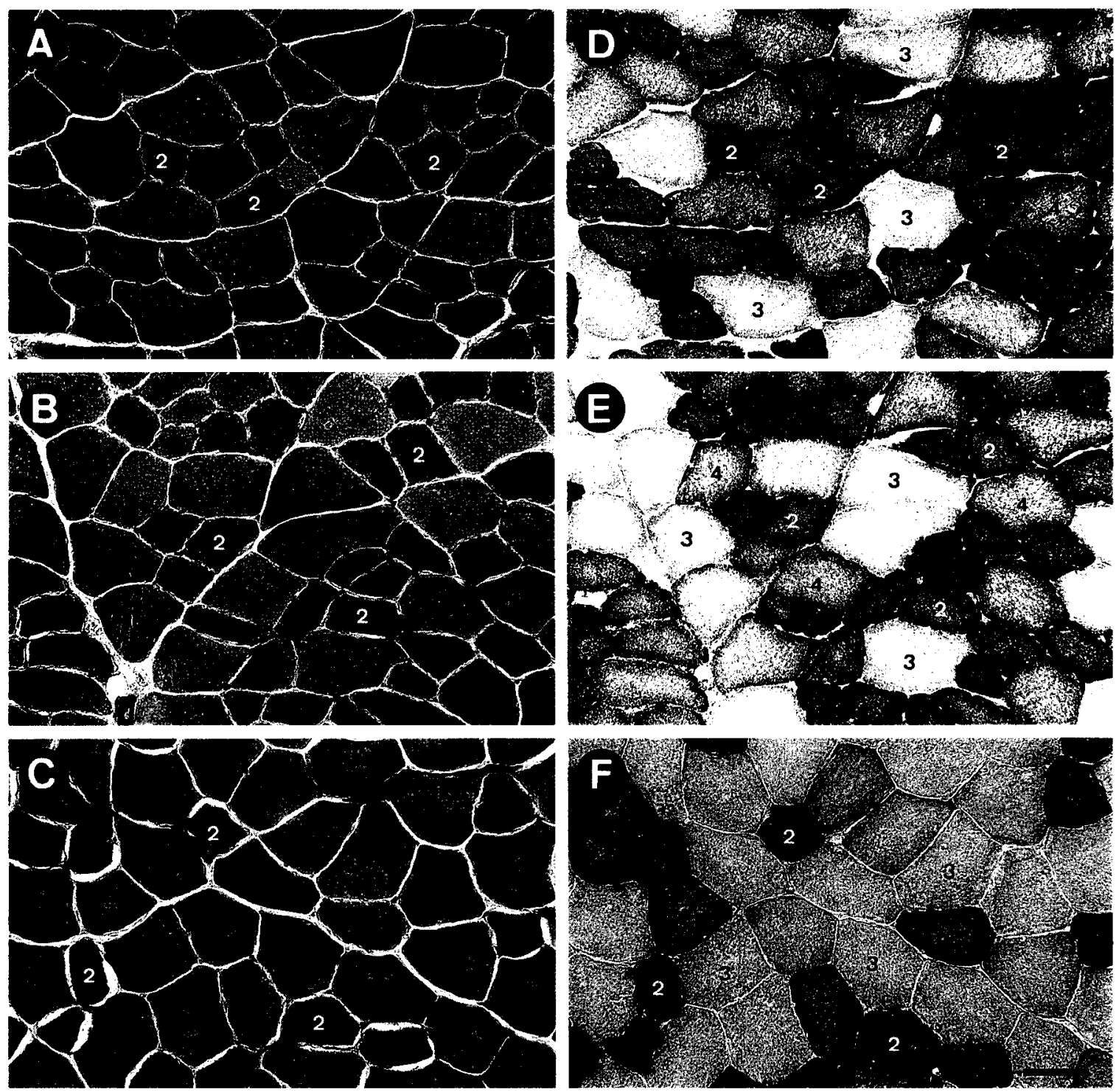

Fig. 3. Serial transverse sections of the deep (A and $\mathbf{D})$, middle $(\mathbf{B}$ and $\mathbf{E})$, and superficial $(\mathbf{C}$ and $\mathbf{F})$ regions of the tibialis anterior muscle in a 10-week-old mouse. The sections are stained for myosin adenosine triphosphatase activity following preincubation at $\mathrm{pH} 4.5(\mathbf{A}, \mathbf{B}$, and $\mathbf{C})$ and for succinate dehydrogenase activity (D, E, and F). 2, type IIa; 3, type IIb; 4, type IIx. Scale bar in F indicates $50 \mu \mathrm{m}$.

stainings for type IIa fibers and negative stainings for type IIb fibers following preincubation at pH 4.5 (unpublished observation). Therefore, type IIa and type IIb fibers in the mouse tibialis anterior muscle were replaced with each other in previous studies $[23,24]$, because muscle fibers were classified based only on differences in the $\mathrm{pH}$ lability of mATPase activity. It is concluded that type IIa fibers have higher oxidative enzyme activities and smaller sizes than type IIb fibers in the mouse tibialis anterior muscle as well as in the rat tibialis anterior muscle.

Previous studies $[7,8,25,26,34]$ demonstrated that muscle fiber types are associated with their sizes, oxidative and/or glycolytic enzyme activities, although other studies $[18,19,22,27,31]$ reported wide variations in the mitochondrial oxidative enzyme activities of fibers in the muscle even within the same fiber type defined by mATPase activities or MHC isoform expressions. In the present study, we have focused on the relationship between cell size and oxidative enzyme activity of the same type of fibers in different regions of the muscle, e.g. the deep, middle, and superficial regions and in different species, e.g. mice and rats.

The present study showed that type IIa, type IIx, and type IIb fibers in the deep region of the rat tibialis anterior muscle had smaller sizes and higher SDH activities than the corresponding type of fibers in the middle and superficial regions. It is concluded that the fibers in the deep region of the muscle have more aerobic capacities com- 

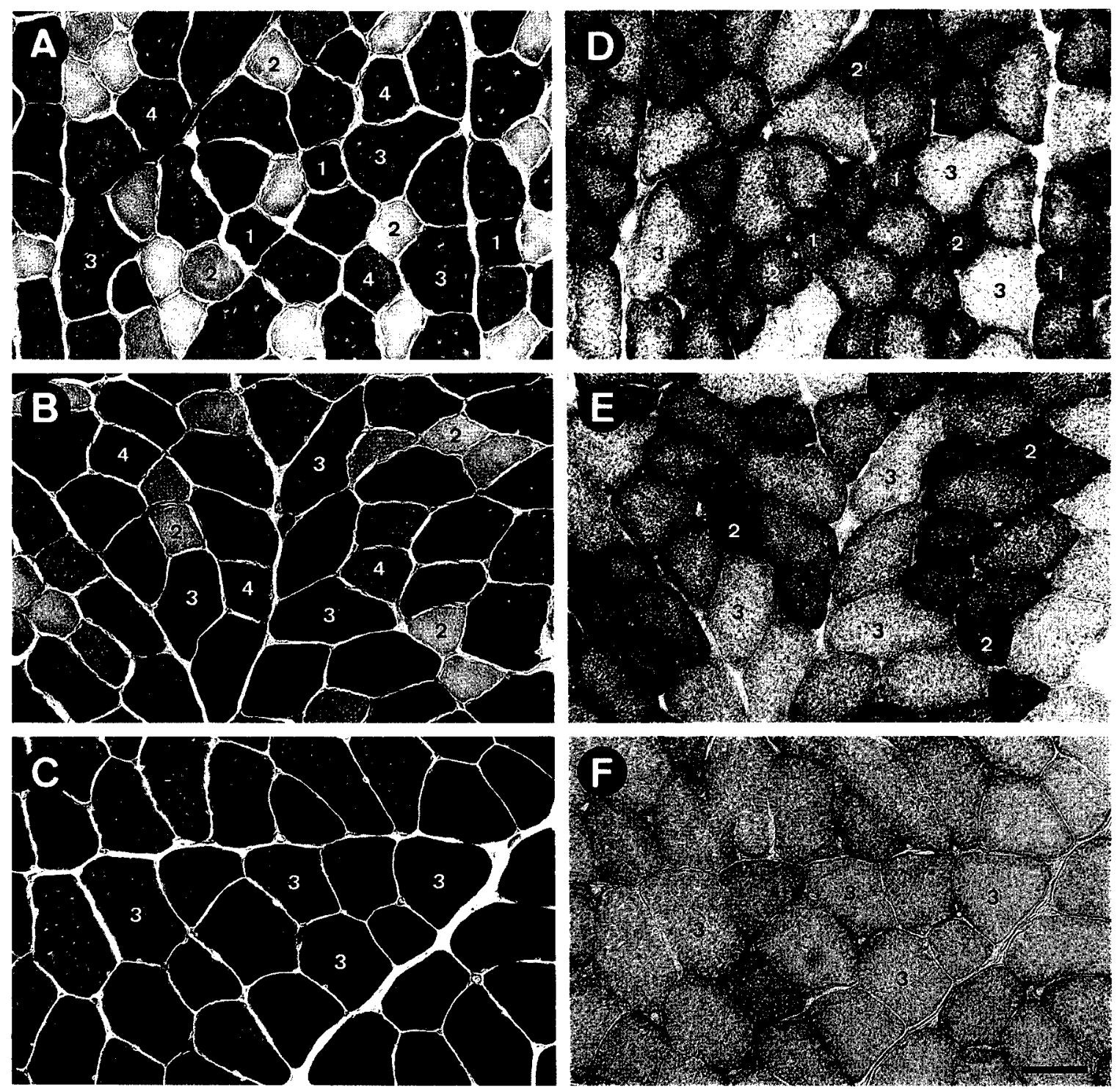

Fig. 4. Serial transverse sections of the deep (A and $\mathbf{D})$, middle (B and $\mathbf{E})$, and superficial $(\mathbf{C}$ and $\mathbf{F})$ regions of the tibialis anterior muscle in a 10-week-old rat. The sections are stained for myosin adenosine triphosphatase activity following preincubation at $\mathrm{pH} 4.5(\mathbf{A}, \mathbf{B}$, and $\mathbf{C})$ and for succinate dehydrogenase activity (D, E, and F). 1, type I; 2, type IIa; 3, type IIb; 4, type IIx. Scale bar in F indicates $50 \mu \mathrm{m}$.

pared with the same type of fibers in the middle and superficial regions, because oxidative metabolism requires a smaller fiber size to reduce the diffusion distance of oxygen and nutrients to the mitochondria in the intermyofibrillar region [35]. It is, therefore, suggested that a differing metabolic demand for fibers depending on the muscle region exists even within the same fiber type. In contrast, there were no regional differences in the SDH activity of type IIa, type IIx, and type IIb fibers in the mouse tibialis anterior muscle, although the CSAs of the fibers were in the rank order of deep $<$ middle $<$ superficial. It is of interest to note that there were no differences in the oxidative enzyme activity of fibers within the same type having different fiber sizes in the mouse muscle. This means that the same type of fibers have similar metabolic demands, irrespective of the muscle region.

In the present study, muscle fibers in mice had higher $\mathrm{SDH}$ activities than the corresponding type of fibers in rats, irrespective of the muscle region. When small and large adults of different species or different sized animals of the same species are compared, the metabolic rate per body weight is inversely related to body size, indicating that the cells and tissues in smaller animals use energy at a higher rate compared with larger ones [30, 32]. These differences in the enzyme activity have already been reported in previous studies using neural cells; the oxidative capacity of spinal neurons varies in an inverse relationship to their body size $[5,12]$. Therefore, a rela- 

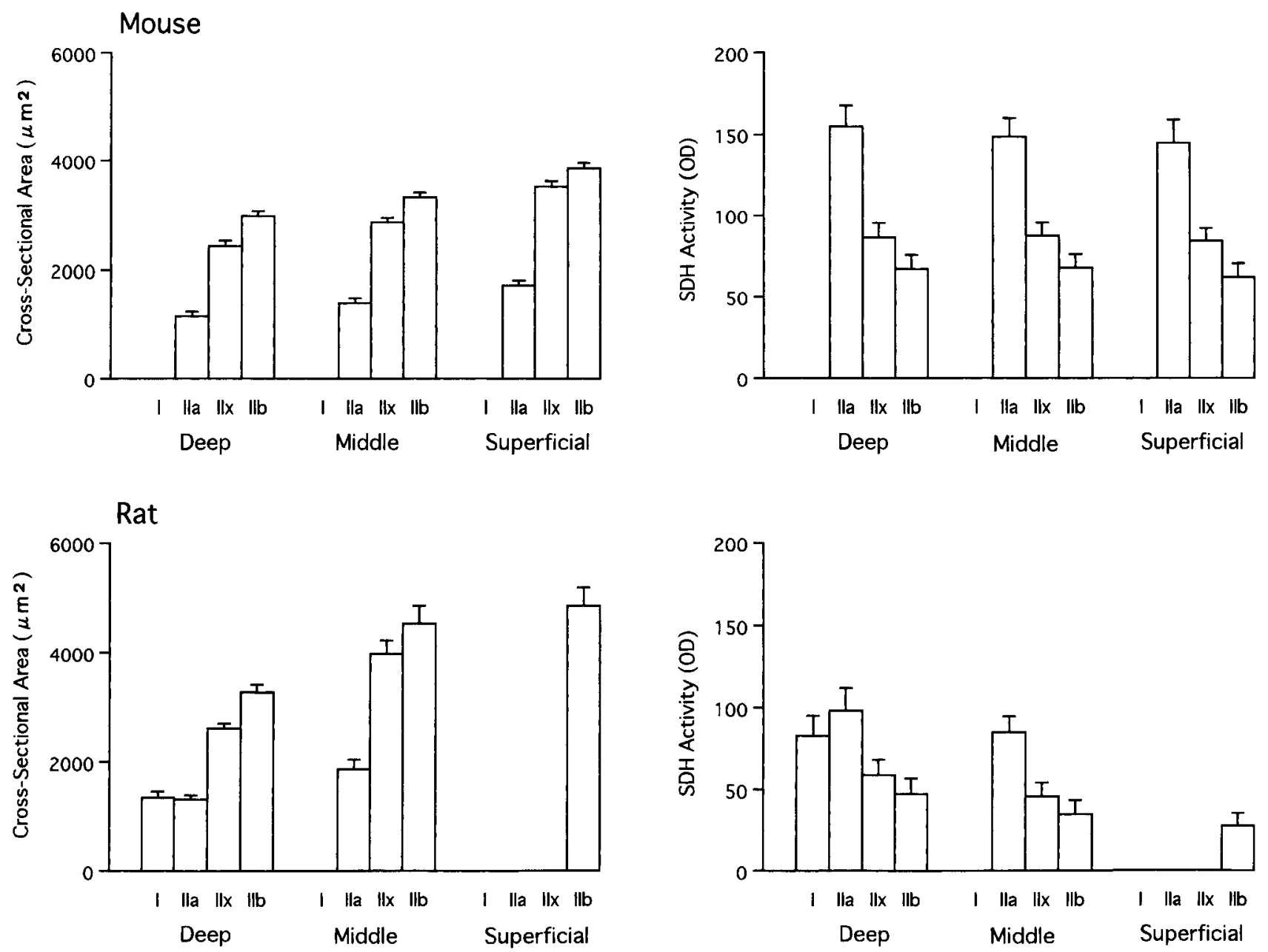

Fig. 5. Mean cross-sectional areas and mean succinate dehydrogenase activities of different types of fibers in the different regions of the mouse (top) and rat (bottom) tibialis anterior muscles. Means and standard deviations from eight animals. SDH, succinate dehydrogenase; OD, optical density. See Tables 1 and 2 for statistical significances.

Table 1. Cross-sectional areas and succinate dehydrogenase activities of different types of fibers in the mouse and rat tibialis anterior muscles.

\begin{tabular}{|c|c|c|}
\hline & $\operatorname{CSA}$ & SDH Activity \\
\hline \multicolumn{3}{|l|}{ Mouse } \\
\hline Deep & $\mathrm{IIa} \ll \ll \operatorname{IIx} \ll \ll \operatorname{IIb}$ & IIa $\gg I I x \gg \gg I I b$ \\
\hline Middle & $\mathrm{IIa} \ll \ll \mathrm{IIx} \ll \ll \mathrm{IIb}$ & IIa $\gg>I I x \gg \gg I I b$ \\
\hline Superficial & IIa $\ll$ IIx $\ll$ IIb & IIa $\gg \gg I I x \gg \gg I I b$ \\
\hline \multicolumn{3}{|l|}{ Rat } \\
\hline Deep & $\mathrm{I}=\mathrm{II} \mathrm{a} \ll \ll \mathrm{IIx} \ll \ll \mathrm{IIb}$ & $\mathrm{IIa}>\mathrm{I} \gg \gg \mathrm{IIx}>\mathrm{IIb}$ \\
\hline Middle & $\mathrm{IIa} \ll \ll \mathrm{IIx} \ll \mathrm{IIb}$ & IIa $\gg>I I x>I I b$ \\
\hline Superficial & - & - \\
\hline
\end{tabular}

$=$, no significance; $>, p<0.05 ; \ll, p<0.01 ; \ll$ and $\gg$, $p<0.001$. There are only type IIb fibers in the superficial region of the rat tibialis anterior muscle. CSA, cross-sectional area; SDH, succinate dehydrogenase.
Table 2. Cross-sectional areas and succinate dehydrogenase activities of fibers in different regions of the mouse and rat tibialis anterior muscles.

\begin{tabular}{|c|c|c|}
\hline & CSA & SDH Activity \\
\hline \multicolumn{3}{|c|}{ Mouse } \\
\hline I & - & - \\
\hline IIa & $\mathrm{D} \ll \ll \mathrm{M} \ll \ll \mathrm{S}$ & $\mathrm{D}=\mathrm{M}=\mathrm{S}$ \\
\hline IIx & $\mathrm{D} \ll \ll \mathrm{M} \ll \ll \mathrm{S}$ & $\mathrm{D}=\mathrm{M}=\mathrm{S}$ \\
\hline $\mathrm{IIb}$ & $\mathrm{D} \ll \mathrm{M} \ll \mathrm{S}$ & $\mathrm{D}=\mathrm{M}=\mathrm{S}$ \\
\hline \multicolumn{3}{|l|}{ Rat } \\
\hline I & - & - \\
\hline IIa & $\mathrm{D} \ll \ll \mathrm{M}$ & $\mathrm{D}>\mathrm{M}$ \\
\hline IIx & $\mathrm{D} \ll \ll$ & $\mathrm{D}>\mathrm{M}$ \\
\hline $\mathrm{IIb}$ & $\mathrm{D} \ll \ll \mathrm{M}=\mathrm{S}$ & $\mathrm{D}>\mathrm{M}=\mathrm{S}$ \\
\hline
\end{tabular}

$=$, no significance; $>, p<0.05 ; \ll, p<0.001$. There are no type I fibers in the mouse tibialis anterior muscle, while type I fibers are observed only in the deep region of the rat tibialis anterior muscle. CSA, cross-sectional area; SDH, succinate dehydrogenase; D, deep region; $\mathrm{M}$, middle region; $\mathrm{S}$, superficial region. 
tively higher activity of the oxidative enzyme in muscle fibers of mice compared to rats would be expected as observed in the present study.

In conclusion, the present data indicate that there are species-specific patterns and regional differences in the CSA and the SDH activity of fibers in the muscle even within the same fiber type defined by MHC isoform expressions that affect the functional role of the whole muscle.

\section{Acknowledgments}

This study was partly supported by a Grant-in-Aid for Scientific Research from the Ministry of Education, Science, and Culture, Japan (09680101, 11680025).

\section{References}

1. Blanco, C. E., Sieck, G. C. and Edgerton, V. R.: Quantitative histochemical determination of succinic dehydrogenase activity in skeletal muscle fibres. Histochem. J. 20; 230-243, 1988.

2. Brooke, M. H. and Kaiser, K. K.: Muscle fiber types: how many and what kind? Arch. Neurol. 23; 369-379, 1970.

3. Brooke, M. H. and Kaiser, K. K.: Three "myosin adenosine triphosphatase" systems: the nature of their $\mathrm{pH}$ lability and sulfhydryl dependence. J. Histochem. Cytochem. 18; 670672, 1970.

4. Chalmers, G. R. and Edgerton, V.R.: Marked and variable inhibition by chemical fixation of cytochrome oxidase and succinate dehydrogenase in single motoneurons. J. Histochem. Cytochem. 37; 899-901, 1989.

5. Chalmers, G. R. and Edgerton, V. R.: Single motoneurons succinate dehydrogenase activity. J. Histochem. Cytochem. 37; 1107-1114, 1989.

6. DeNardi, C., Ausoni, S., Moretti, P., Gorza, L., Velleca, M., Buckingham, M. and Schiaffino, S.: Type 2X-myosin heavy chain is coded by a muscle fiber type-specific and developmentally regulated gene. J. Cell Biol. 123; 823-835, 1993.

7. Dix, D. J. and Eisenberg, B. R.: Expression of a fast myosin heavy chain mRNA in individual rabbit skeletal muscle fibers with intermediate oxidative capacity. Anat. Rec. 230; 52-56, 1991.

8. Hämäläinen, N. and Pette, D.: The histochemical profiles of fast fiber types IIB, IID, and IIA in skeletal muscles of mouse, rat, and rabbit. J. Histochem. Cytochem. 41; 733-743, 1993.

9. Hirofuji, C., Ishihara, A., Roy, R. R., Itoh, K., Itoh, M., Edgerton, V. R. and Katsuta, S.: SDH activity and cell size of tibialis anterior motoneurons and muscle fibers in SAMP6. Neuroreport 11; 823-828, 2000.

10. Inoue, K., Tamada, Y., Munekawa, K., Hayashi, S., Iijima, N., Ishihara, A., Ikeda, T. and Tanaka, M.: Astroglial elements in the suprachiasmatic nucleus of the golden hamster and the rat -comparative morphological study using immunohistochemistry-. Acta Histochem. Cytochem. 33; 95-102, 2000.

11. Ishihara, A., Roy, R. R. and Edgerton, V. R.: Succinate dehydrogenase activity and soma size of motoneurons innervating different portions of the rat tibialis anterior. Neuroscience 68; 813-822, 1995.

12. Ishihara, A., Roy, R. R. and Edgerton, V.R.: Comparison of succinate dehydrogenase activity and soma size relationships among neurons in dorsal root ganglia of rats and cats. Brain Res. 716; 183-186, 1996.

13. Izumo, S., Nadal-Ginard, B. and Mahdavi, V.: All members of the MHC multigene family respond to thyroid hormone in a highly tissue-specific manner. Science 231; 597-600, 1986.

14. Larsson, L., Edström, L., Lindergren, B., Gorza, L. and Schiaffino, S.: MHC composition and enzyme-histochemical and physiological properties of a novel fast-twitch motor unit type. Am. J. Physiol. 261; C93-C101, 1991.

15. Martin, T. P., Vailas, A. C., Durivage, J. B., Edgerton, V. R. and Castleman, K. R.: Quantitative histochemical determination of muscle enzymes. J. Histochem. Cytochem. 33; 1053$1059,1985$.

16. Nakatani, T., Nakashima, T., Kita, T., Hirofuji, C., Itoh, K., Itoh, M. and Ishihara, A.: Succinate dehydrogenase activities of fibers in the rat extensor digitorum longus, soleus, and cardiac muscles. Arch. Histol. Cytol. 62; 393-399, 1999.

17. Nakatani, T., Nakashima, T., Kita, T., Hirofuji, C., Itoh, K., Itoh, M. and Ishihara, A.: Cell size and oxidative enzyme activity of different types of fibers in different regions of the rat plantaris and tibialis anterior muscles. Jpn. J. Physiol. (in press)

18. Nemeth, P. and Pette, D.: Succinate dehydrogenase activity in fibres classified by myosin ATPase in three hind limb muscles of rat. J. Physiol. (Lond.) 320; 73-80, 1981.

19. Pette, D.: Microphotometric measurement of initial maximum reaction rates in quantitative enzyme histochemistry in situ. Histochem. J. 13; 319-327, 1981.

20. Pool, Chr. W., Diegenbach, P. C. and Scholten, G.: Quantitative succinate-dehydrogenase histochemistry. I. Methodological study on mammalian and fish muscle. Histochemistry 64; 251-262, 1979.

21. Pool, Chr. W., Diegenbach, P. C. and Ockeloen, B. J. T.: Quantitative succinate-dehydrogenase histochemistry. II. A comparison between visual and quantitative muscle fibre typing. Histochemistry 64; 263-272, 1979.

22. Reichmann, H.: Quantitative enzyme histochemistry in muscle fibres. Acta Histochem. (Suppl.) 37; 45-51, 1989.

23. Reichmann, H. and Pette, D.: A comparative microphotometric study of succinate dehydrogenase activity levels in type I, IIA and IIB fibres of mammalian and human muscles. Histochemistry 74; 27-41, 1982.

24. Reichmann, H. and Pette, D.: Glycerolphosphate oxidase and succinate dehydrogenase activities in IIA and IIB fibres of mouse and rabbit tibialis anterior muscles. Histochemistry 80 ; 429-433, 1984.

25. Rivero, J.-L. L., Talmadge, R. J. and Edgerton, V. R.: Fibre size and metabolic properties of myosin heavy chain-based fibre types in rat skeletal muscle. J. Muscle Res. Cell Motil. 19; 733-742, 1998.

26. Rivero, J.-L. L., Talmadge, R. J. and Edgerton, V. R.: Interrelationships of myofibrillar ATPase activity and metabolic properties of myosin heavy chain-based fibre types in rat skeletal muscle. Histochem. Cell Biol. 111; 277-287, 1999.

27. Rosser, B. W. C., Norris, B. J. and Nemeth, P. M.: Metabolic capacity of individual muscle fibers from different anatomic locations. J. Histochem. Cytochem. 40; 819-825, 1992.

28. Schiaffino, S. and Reggiani, C.: Molecular diversity of myofibrillar proteins: gene regulation and functional significance. Physiol. Rev. 76; 371-423, 1996.

29. Schiaffino, S., Gorza, L., Sartore, S., Saggin, L., Ausoni, S., Vianello, M., Gundersen, K. and Lømo, T.: Three myosin heavy chain isoforms in type 2 skeletal muscle fibres. J. Muscle Res. Cell Motil. 10; 197-205, 1989.

30. Schmidt-Nielsen, K.: Locomotion energy cost of swimming, flying, and running. Science 177; 222-228, 1972.

31. Sickles, D. W., McLendon, R.E. and Rosenquist, ThH.: Alternative method for quantitative enzyme histochemistry of muscle fibers. Application of photographic densitometry 
combined with atomic absorption spectrophotometry. Histochemistry 73; 577-588, 1982.

32. Taylor, C. R., Schmidt-Nielsen, K. and Raab, J. L.: Scaling of energetic cost of running to body size in mammals. $A m$. $J$. Physiol. 219; 1104-1107, 1970.

33. Termin, A., Staron, R. S. and Pette, D.: Myosin heavy chain isoforms in histochemically defined fiber types of rat muscle.
Histochemistry 92; 453-457, 1989.

34. Wank, V., Bauer, R., Punkt, K. and Ziegan, J.: Enzyme activity patterns of myosin ATPase, $\alpha$-glycerophosphate dehydrogenase and succinate dehydrogenase within different muscle fibre types. Acta Histochem. 96; 213-218, 1994.

35. Wittenberg, B. A. and Wittenberg, J. B.: Transport of oxygen in muscle. Annu. Rev. Physiol. 51; 857-878, 1989. 